\title{
El riesgo de infecciones respiratorias en el fumador activo y pasivo
}

\author{
FERNANDO SALDÍAS P.*, J. IGNACIO MÉNDEZ C.**, \\ DAVID RAMÍREZ R.** y ORLANDO DÍAZ P.*
}

\section{Tobacco smoke and risk of respiratory infection}

Tobacco smoke is a proven risk factor for viral and bacterial respiratory infection. In adults without COPD, smoking is associated with a significant increase in the relative risk of pneumonia $(O R=2.0 ; 95 \%$ CI 1.24-3.24), invasive pneumococcal disease $(O R=2.6 ; 95 \%$ CI 1.9-3.5) and Legionella infection (OR $=3.48 ; 95 \%$ CI 2.09-5.79). Smoking has clearly been shown to be associated with an increased risk of influenza $(O R=2.4$; 95\% CI 1.5-3.8), tuberculosis $(O R=2.6$; $95 \%$ CI 2.2-3.1) and varicella pneumonia. In young children whose parents smoke, passive exposure to tobacco smoke is associated with an increased relative risk of seasonal infections (bronchitis, pneumonia) $(O R=1.72$; CI 95\% 1.55-1.91) and recurrent otitis media $(O R=1.88 ; 95 \%$ CI 1.023.49). Passive smoking also increases risk of pneumonia in adults (OR $=2.5 ;$ CI 95\% 1.2-5.1). Plausible explanations of the increased risk of infection in active or passive smokers include increased bacterial adherence, decrease of lung and nasal clearance, and changes in the immune response. Conclusions: Exposure to tobacco smoke approximately doubles the risk of infection. This increased burden of infection has significant healthcare cost implications. Each infectious episode in an individual should prompt an attempt at smoking cessation.

Key words: respiratory infection, influenza, tuberculosis, pneumonia, risk factors, smoking.

\section{Resumen}

La exposición a humo de tabaco constituye un importante factor de riesgo para adquirir infecciones respiratorias bacterianas y virales. En adultos sin enfermedad pulmonar obstructiva crónica, el tabaquismo está asociado a un aumento significativo del riesgo de neumonía (OR: 2,0; IC95\%: 1,24-3,24), enfermedad neumocócica invasiva (OR: 2,6; IC95\%: 1,9-3,5) e infección por Legionella spp. (OR: 3,48; IC95\%: 2,09-5,79). Además, el tabaquismo está asociado a mayor riesgo de influenza (OR: 2,4; IC95\%: 1,5-3,8), tuberculosis (OR: 2,6; IC95\%: 2,2-3,1) y neumonía por virus varicela. En niños pequeños expuestos al humo de tabaco en sus hogares aumenta el riesgo de infecciones respiratorias bajas (bronquitis, neumonía) (OR: 1,72; IC95\%: 1,55-1,91) y otitis media recurrente (OR: 1,88; IC95\%: 1,02-3,49). El tabaquismo pasivo también aumenta el riesgo de neumonía en adultos (OR: 2,5; IC95\%: 1,2-5,1). El aumento del riesgo de infecciones respiratorias en el fumador activo y pasivo puede ser parcialmente atribuido al aumento de la adherencia y colonización bacteriana de la mucosa respiratoria, disminución de la depuración mucociliar nasal y de la vía aérea, y alteraciones específicas de la inmunidad humoral y celular. Conclusión: La exposición a humo de tabaco aumenta al doble el riesgo de infecciones respiratorias en niños y adultos. El aumento de las consultas por infecciones respiratorias constituye una elevada carga para el sistema de salud. En los pacientes que consultan por infecciones respiratorias agudas se debería evaluar el riesgo individual de exposición a humo de tabaco y el equipo de salud debería implementar actividades educativas específicas para intentar controlarlo.

Palabras clave: infección respiratoria, influenza, tuberculosis, neumonía, factores de riesgo, tabaquismo.

\footnotetext{
* Departamento de Enfermedades Respiratorias, Pontificia Universidad Católica de Chile.

** Internos de Medicina, Pontificia Universidad Católica de Chile.
} 


\section{Introducción}

El tabaquismo es el principal factor de riesgo de muerte prematura prevenible en el mundo ${ }^{1}$. En la actualidad (2004), la Organización Mundial de la Salud estima que ocurren 4,9 millones de muertes anuales relacionadas con el consumo de tabaco. El consumo activo y pasivo de tabaco tiene efectos deletéreos sobre múltiples órganos, especialmente el sistema respiratorio ${ }^{2}$. El cáncer bronquial y la enfermedad pulmonar obstructiva crónica son las principales enfermedades respiratorias asociadas al tabaquismo ${ }^{3}$. En las últimas décadas, se ha reconocido la importancia del tabaquismo pasivo en la salud del niño ${ }^{4}$.

La exposición prolongada a humo de tabaco también afecta la incidencia, gravedad y evolución de múltiples otras afecciones respiratorias, como resfrío común, influenza, neumonía, tuberculosis, neumotórax, hemorragia pulmonar y algunas enfermedades intersticiales ${ }^{5}$. Los mecanismos patogénicos por los cuales el consumo de tabaco aumenta la incidencia y gravedad de algunas enfermedades respiratorias no han sido completamente esclarecidos. El tabaquismo activo y la exposición a humo de tabaco (fumador pasivo) aumentan significativamente el riesgo de infecciones respiratorias, lo cual no es ampliamente reconocido por el equipo médico ${ }^{5}$. En este artículo revisaremos los trastornos fisiológicos y morfológicos ocasionados por el consumo de tabaco sobre el sistema respiratorio y el sistema inmune, que podrían explicar el aumento del riesgo de padecer infecciones respiratorias en la etapa infantil y adulta.

\section{Efectos del tabaquismo en el aparato respiratorio y sistema inmune}

Los mecanismos patogénicos que explican el aumento del riesgo de infecciones sistémicas en sujetos expuestos al humo de tabaco han sido sólo parcialmente esclarecidos (Tabla 1). Ellos son multifactoriales y probablemente interactúan en sus efectos fisiológicos, existiendo mecanismos estructurales e inmunológicos.

El consumo de tabaco ocasiona cambios patológicos en virtualmente todos los componentes del aparato respiratorio. La exposición activa o pasiva a humo de tabaco ocasiona los siguientes cambios morfológicos en el pulmón: inflamación y fibrosis peribronquiolar, alteración de la estructura y función del epitelio alveolar, engrosamiento de la íntima vascular y destrucción de alvéolos ${ }^{6}$. Las alteraciones funcionales asociadas al tabaquismo incluyen la disminución del clearance mucociliar de sustancias inhaladas, favorece la adherencia de microorganismos a la mucosa respiratoria y cambios en la permeabilidad alvéolo-capilar secundario al aumento en el número y actividad de las células inflamatorias en el pulmón ${ }^{7-9}$.

El lavado broncoalveolar (LBA) ha permitido examinar los cambios inflamatorios e inmunológicos que acontecen en el pulmón asociado al tabaquismo ${ }^{10}$. En los fumadores se eleva en forma significativa el recuento celular, especialmente el recuento de polimorfonucleares y macrófa$\operatorname{gos}^{11}$. El recuento de linfocitos se mantiene inalterable pero aumenta el porcentaje de células $\mathrm{CD} 4+$ y la relación $\mathrm{CD} 4 / \mathrm{CD} 8{ }^{10}$.

Tabla 1. Efectos sobre el sistema respiratorio de los principales componentes químicos del humo de cigarrillo

\begin{tabular}{ll}
\hline Productos químicos & \multicolumn{1}{c}{ Efectos sobre el sistema respiratorio } \\
\hline Monóxido de carbono & Inhibe el transporte de oxígeno a los tejidos \\
Dióxido de carbono & Depresor respiratorio \\
Oxido de nitrógeno & Irritante respiratorio \\
Formaldehído & Irritante respiratorio, alteración de la función mucociliar \\
Dimetilnitrosamina & Carcinógeno \\
Dióxido de sulfuro & Irritante respiratorio, deterioro de la función mucociliar \\
Nicotina & Irritante respiratorio, deterioro de la función mucociliar, depresor de la respuesta \\
& inmune \\
Benzopireno & Carcinógeno \\
Acroleína & Irritante respiratorio, ciliostático \\
Amonio & Irritante respiratorio y ocular \\
Compuestos inorgánicos & Carcinógenos, efectos tóxicos directos sobre el sistema respiratorio \\
(plomo, níquel, cadmio) & \\
\hline
\end{tabular}


El tabaquismo afecta la morfología y función de las células inflamatorias del pulmón. En los fumadores, los macrófagos alveolares son más grandes y presentan cambios en su superficie e inclusiones citoplasmáticas que pueden deteriorar su capacidad de presentación de antígenos ${ }^{12}$. Se ha observado un aumento, relacionado con la dosis, en la concentración de citoquinas proinflamatorias (IL-1 $\beta$ e IL-8), y la respuesta de citoquinas de los macrófagos a variados estímulos puede estar alterada ${ }^{11,13,14}$. En los fumadores, la quimiotaxis y migración de los neutrófilos desde el espacio vascular está disminuida asociado a su activación en la circulación pulmonar ${ }^{15}$. La respuesta a mitógenos de los linfocitos del pulmón está disminuida en fumadores, lo cual no ocurre con los linfocitos de sangre periférica ${ }^{16}$. En los fumadores disminuyen los niveles de inmunoglobulinas séricas y en el líquido de LBA, que pueden persistir después de haber suspendido el consumo de tabaco ${ }^{10}$.

En los fumadores, el recuento de neutrófilos en sangre periférica está aumentado, lo cual es mediado por estimulación de la hematopoyesis y acortamiento del tiempo de permanencia en la médula ósea ${ }^{17}$. Otras subpoblaciones de leucocitos también aumentan en sangre periférica, como ocurre con el recuento de linfocitos $\mathrm{T}$ supresores $(\mathrm{CD} 8+)^{18}$. Además, se ha observado un descenso reversible de la actividad de las células natural killer en los fumadores activos ${ }^{19}$, las cuales estarían involucradas en la respuesta inmune precoz contra las infecciones virales.

El aparato respiratorio de los fumadores está expuesto a un mayor estrés oxidativo determinado por las sustancias oxidantes contenidas en el humo de cigarrillo y aquellas liberadas por la activación de las células inflamatorias residentes en el pulmón ${ }^{20}$. Aunque la exposición aguda a agentes oxidantes ocasiona incremento en la producción de sustancias antioxidantes a nivel celular, los niveles de antioxidantes séricos de los fumadores están disminuidos ${ }^{21}$. El desbalance en la actividad oxidante y antioxidante puede contribuir al daño pulmonar de los fumadores.

La exposición prolongada a humo de tabaco activa y pasiva aumenta el riesgo de infecciones del tracto respiratorio superior e inferior, tales como el resfrío común, otitis media, infecciones por virus influenza y respiratorio sincicial, bronquitis, neumonías y otras infecciones bacterianas graves (infección neumocócica invasiva y meningococcemia) $)^{5,22-27}$. Los principales mecanismos involucrados en el aumento del riesgo de infecciones en el fumador activo y pasivo son:

\section{Cambios estructurales}

a) Daño anatómico en el tracto respiratorio: El humo de tabaco y muchos de sus componentes ocasionan cambios estructurales en el aparato respiratorio (inflamación y fibrosis peribronquiolar, disminución del clearance mucociliar y disrupción del epitelio respiratorio) que predisponen al desarrollo de infecciones respiratorias $^{6,28}$. Varios componentes del humo de tabaco, incluyendo la acroleína, acetaldehído, formaldehído, radicales libres producto de reacciones químicas y óxido nítrico pueden contribuir al daño estructural y funcional del epitelio respiratorio.

b) Adherencia bacteriana a la mucosa respiratoria: La colonización e infección por microorganismos a menudo requiere la adherencia selectiva a las células epiteliales del huésped. Varios estudios demuestran que la nicotina ocasiona daño tóxico directo sobre el epitelio respiratorio y aumenta la adherencia de bacterias patógenas a la mucosa respiratoria ${ }^{28}$. La colonización de la vía aérea por $S$. pneumoniae, $H$. influenzae y $M$. catarrhalis en sujetos fumadores explicaría en parte su mayor susceptibilidad a padecer infecciones respiratorias.

\section{Cambios inmunológicos}

a) Respuesta inmune inespecífica: En los fumadores, el recuento de leucocitos en sangre periférica es $30 \%$ más elevado que en los sujetos no fumadores, lo cual ha sido atribuido a la liberación de catecolaminas o estimulación de la médula ósea mediado por nicotina u otros componentes del humo de tabaco ${ }^{17,18}$. La nicotina inhibe la actividad fagocitaria de los neutrófilos y monocitos-macrófagos disminuyendo la producción de radicales de oxígeno ${ }^{29}$. Los aldehídos insaturados del humo de tabaco inhiben la quimiotaxis de los polimorfonucleares ${ }^{30}$. La actividad fagocítica de los macrófagos alveolares está claramente disminuida en los sujetos fumadores, lo cual es confirmado por los estudios experimentales $^{31}$. La liberación de citoquinas de los macrófagos está disminuida en los fumadores, especialmente la producción de interleuquina (IL) 1, IL-2, IL-6, TNF- $\alpha$ e interferón $\gamma^{12-14}$. Estudios en animales han mostrado que el déficit de IL 1 e IL 6 aumentan el riesgo de desarrollar neumonía bacteriana. El trastorno funcional de los polimorfonucleares ocasionado por el tabaco puede contribuir al mayor riesgo de infecciones sistémicas en los fumadores, incluyendo la neumonía bacteriana.

b) Respuesta inmune específica: Se han empleado anticuerpos monoclonales para examinar 
los cambios en las subpoblaciones de linfocitos $\mathrm{T}$ en la sangre periférica determinados por la exposición a humo de tabaco. En los fumadores activos ( $\geq 50$ paquetes/año) disminuye el recuento sanguíneo de linfocitos CD4+ y aumentan los linfocitos CD8+, siendo este efecto reversible al dejar de fumar ${ }^{32}$. La nicotina inhibe la respuesta celular Th 1 (responsable de la producción de inmunoglobulinas) y estimula selectivamente la respuesta Th 2 y la producción de algunas citoquinas, como la IL-4, IL-5, IL-10 e IL-13 (responsable de las manifestaciones clínicas asociadas a algunas enfermedades atópicas como la rinitis, eczema y asma bronquial $^{33}$. La nicotina estimula los eosinófilos y la producción de inmunoglobulina $\mathrm{E}$ a partir de los linfocitos B. Mientras que la supresión de la respuesta Th 1 por nicotina, disminuye la producción de inmunoglobulinas tipo $\operatorname{IgA}$ e $\operatorname{IgG}_{2}$, sin modificar la síntesis de $\operatorname{IgM}^{34}$. La disminución en el recuento sanguíneo y lavado broncoalveolar de células $\mathrm{CD} 4+$, que facilitan la proliferación y diferenciación de los linfocitos B y la síntesis de inmunoglobulinas, puede contribuir al mayor riesgo de infecciones en los fumadores.

En resumen, la exposición a humo de tabaco ocasiona variadas alteraciones en la respuesta inmune celular y humoral de los individuos, incluyendo una disminución en los niveles circulantes de inmunoglobulinas, inhibición de la respuesta de anticuerpos a ciertos antígenos, disminución del recuento sanguíneo y pulmonar de linfocitos CD4+ y aumento de CD8+, disminución de la actividad fagocitaria y liberación de citoquinas proinflamatorias (Tabla 2). Es impor- tante reconocer que muchas de las alteraciones inmunológicas observadas en los fumadores son rápidamente reversibles y se resuelven después de seis semanas de haber dejado de fumar, lo cual sustenta la idea que la suspensión del hábito tabáquico constituye una medida efectiva para prevenir el desarrollo de infecciones.

\section{Tabaquismo e infecciones respiratorias}

\section{Resfrío común}

Varios estudios epidemiológicos sugieren que los fumadores tendrían mayor número de resfríos en el año y éstos serían más severos ${ }^{35-38}$. Peters y Ferris $^{35}$, comunicaron mayor número de consultas por síntomas respiratorios agudos compatibles con resfrío en escolares fumadores, lo cual ha sido confirmado por otros autores ${ }^{36}$. Otro estudio realizado en la comunidad encontró que los fumadores era más probable que refirieran síntomas del tracto respiratorio inferior, que estos síntomas fueran más prolongados y presentaran mayores anomalías en la auscultación pulmonar ${ }^{37}$. Cohen y cols, expusieron a alrededor de 400 sujetos sanos a virus respiratorios en las narinas, observando en los fumadores mayor riesgo de infección objetivado por cultivo viral o producción de anticuerpos séricos y síntomas de resfrío (odds ratio (OR): 2,23; IC95\%: 1,03-4,82) ${ }^{38}$. Este efecto del tabaco ha sido atribuido a un defecto en la respuesta inmune que limita la replicación viral o aumento del proceso inflamatorio que determina la producción de síntomas.

Tabla 2. Efectos de la exposición a humo de tabaco en el sistema inmune

\begin{tabular}{|c|c|c|}
\hline Tipo de células & Efecto & Mecanismo de acción \\
\hline $\begin{array}{l}\text { Macrófagos y } \\
\text { monocitos }\end{array}$ & Inhibición de la inflamación y fagocitosis & $\begin{array}{l}\text { Inhibición anión superóxido, } \mathrm{H}_{2} \mathrm{O}_{2} \\
\text { y producción de radicales de } \mathrm{O}_{2}\end{array}$ \\
\hline Neutrófilos & Inhibición de la inflamación y fagocitosis & $\begin{array}{l}\text { Inhibición anión superóxido, } \mathrm{H}_{2} \mathrm{O}_{2} \\
\text { y producción de radicales de } \mathrm{O}_{2}\end{array}$ \\
\hline Linfocitos $\mathrm{T}$ & $\begin{array}{l}\text { Aumento de la producción de IL-4, IL-5, } \\
\text { IL-10 e IL-13 }\end{array}$ & $\begin{array}{l}\text { Inhibición de la respuesta Th } 1 \\
\text { y estimulación de respuesta Th } 2\end{array}$ \\
\hline Linfocitos B & $\begin{array}{l}\text { Disminución de síntesis Ig } \\
\text { (especialmente IgA e IgG) }\end{array}$ & $\begin{array}{l}\text { Inhibición función de linfocitos B } \\
\text { por supresión de la respuesta Th } 1\end{array}$ \\
\hline Células "natural killer" & $\begin{array}{l}\text { Inhibición o supresión de la actividad } \\
\text { citotóxica }\end{array}$ & Inhibición células natural killer \\
\hline Epitelio respiratorio & $\begin{array}{l}\text { Colonización por bacterias patógenas } \\
\text { de la mucosa respiratoria }\end{array}$ & $\begin{array}{l}\text { Aumento de la adherencia de las bacterias } \\
\text { al epitelio respiratorio }\end{array}$ \\
\hline Función mucociliar & $\begin{array}{l}\text { Inhibición de la depuración mucociliar } \\
\text { de la vía aérea }\end{array}$ & $\begin{array}{l}\text { Daño tóxico directo y disrupción del } \\
\text { epitelio ciliado respiratorio }\end{array}$ \\
\hline
\end{tabular}




\section{Otitis media e infección respiratoria baja}

Existe una relación bien establecida entre el tabaquismo parental y el riesgo de infecciones respiratorias bajas en infantes. Estudios realizados en el hospital y la comunidad muestran que los hijos pequeños de padres fumadores tienen el doble de riesgo de padecer infecciones respiratorias bajas (OR: 1,72; IC95\%: 1,55-1,91) El riesgo relativo es 1,57 (IC95\%: 1,42-1,74) cuando uno de los padres fuma y asciende a 1,72 (IC95\%: 1,55-1,91) cuando es la madre quien fuma en el hogar. Esta asociación se mantiene cuando se controla por otras variables confundentes, y la mayoría de los estudios han demostrado que existe una relación dosis-efecto. Los niños asmáticos y portadores de enfermedades respiratorias crónicas serían más susceptibles a este daño.

Existe una relación entre el tabaquismo parental y el riesgo de otitis en el niño pequeño. La nicotina y otros productos del humo de tabaco favorecen la invasión del oído medio por microorganismos que colonizan la nasofaringe ${ }^{22,40}$. La exposición prolongada a humo de tabaco se ha asociado a mayor riesgo de otitis media recurrente en niños pequeños (OR: 1,88; IC95\%: $1,02-3,49)^{41}$. Parece existir una relación dosisefecto en el riesgo de otitis recurrente. Los principales mecanismos involucrados en este proceso serían la inflamación y congestión de la vía aérea superior e inferior, disfunción mucociliar y bloqueo de la trompa de Eustaquio, aumento de la adherencia de microorganismos al epitelio respiratorio y disminución de la respuesta inmune local (producción de IgA).

\section{Enfermedad periodontal}

En el fumador activo aumenta 2,5 a 6 veces el riesgo de desarrollar enfermedad periodontal comparado con los no fumadores (OR: 2,79; IC95\%: 1,9-4,1 para fumadores $\leq 9$ cigarrillos/día y OR: 5,88 ; IC95\%: 4,0-8,5 para fumadores $\geq 31$ ci-

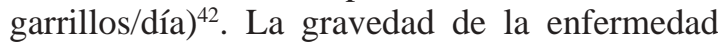
periodontal es mayor en los fumadores y la respuesta al tratamiento quirúrgico es más pobre ${ }^{43}$. El riesgo disminuye progresivamente en el curso de los años al suspender el consumo de tabaco.

\section{Influenza}

Varios estudios han examinado la relación entre tabaquismo e infección por virus influenza ${ }^{44-46}$. En una cohorte de 250 mil veteranos americanos, Rogot y Murray ${ }^{44}$, encontraron en los fumadores un aumento de la mortalidad por influenza (OR: 1,78). Otros han confirmado ha- llazgos similares en sujetos más jóvenes sin factores confundentes, en reclutas israelíes se han reportado episodios más frecuentes y graves de influenza en los fumadores (OR: 2,42; IC95\%: $1,5-3,8)$, y el $31,2 \%$ de los casos de influenza han sido atribuidos al consumo de tabaco ${ }^{45}$. En escolares que fuman se han encontrado mayores tasas de infección por virus influenza y mayor incidencia de infección subclínica objetivada mediante determinación de anticuerpos séricos ${ }^{46}$. En los fumadores, los títulos de anticuerpos séricos disminuyen más rápidamente, cambios inmunológicos que pueden explicar al menos parcialmente la mayor susceptibilidad a la infección viral $^{47}$. La eficacia de la vacuna antigripal sería superior en los sujetos que fuman, porque estarían expuestos a un mayor riesgo de infección que los no fumadores.

\section{Tuberculosis}

El consumo de tabaco se ha asociado a mayor incidencia de reacción de tuberculina (PPD) positiva y tuberculosis activa ${ }^{48-52}$. En un estudio de caso y control en presidiarios, los fumadores presentaron mayor riesgo de viraje tuberculínico en la cárcel comparado con los no fumadores (OR: 1,78), especialmente aquellos que fumaron por tiempos más prolongados ${ }^{48}$. En otro estudio de caso y control, los niños expuestos pasivamente a humo de cigarrillo que eran contacto de un paciente con tuberculosis pulmonar activa presentaban mayor riesgo de desarrollar tuberculosis activa (OR: 5,39; IC95\%: 2,4-11,9) ${ }^{49}$. Además, existe una fuerte asociación entre tabaquismo activo y el desarrollo de tuberculosis pulmonar en adolescentes y adultos jóvenes que cohabitan con un enfermo de tuberculosis (OR: 3,8 para los fumadores activos ajustado para los factores confundentes) $)^{50}$. Varios estudios epidemiológicos poblacionales realizados en el Reino Unido, India y China han comunicado hallazgos similares: el tabaquismo activo y pasivo se asocian a mayor riesgo de desarrollar tuberculosis $\operatorname{activa}^{51,52}$. La incidencia de tuberculosis aumenta 2-4 veces en los fumadores activos (> 20 cigarrillos/día) comparado con el grupo que nunca ha fumado (Tabla 3 ).

Se ha demostrado que el tratamiento inmunosupresor y el empleo de anticuerpos monoclonales contra el factor de necrosis tumoral alfa (TNF$\alpha)$ en enfermedades inflamatorias crónicas como la artritis reumatoidea aumentan el riesgo de reactivación de la tuberculosis, implicando a los linfocitos $\mathrm{T}$ y macrófagos pulmonares que expresan TNF- $\alpha$ en su membrana como elementos protectores en la mantención del estado de 
Tabla 3. Riesgo relativo de enfermedad tuberculosa en población adulta fumadora de la India ${ }^{52}$

\begin{tabular}{lccc}
\hline Consumo de tabaco & Casos/Población & Riesgo relativo & IC95\% \\
\hline No fumador & $355 / 145.665$ & 1 & \\
$<10$ cigarrillos/día & $103 / 22.039$ & 1,7 & $1,4-2,2$ \\
$\geq 10$ cigarrillos/día & $267 / 38.317$ & 2,6 & $2,2-3,1$ \\
$<15$ bidis/día & $125 / 12.563$ & 2,9 & $2,4-3,6$ \\
$\geq 15$ bidis/día & $170 / 10.553$ & 4,5 & $3,7-5,5$ \\
\hline
\end{tabular}

Nota: los bidis son cigarrillos elaborados en forma artesanal por la población hindú

Tabla 4. Riesgo relativo de contraer neumonía en sujetos adultos fumadores. Estudio de caso y control $^{61}$

\begin{tabular}{lccc}
\hline Consumo de tabaco & Pacientes/Control & Riesgo relativo & IC95\% \\
\hline No fumador & $72 / 208$ & 1,00 & \\
1 - 9 cigarrillos/día & $25 / 83$ & 1,24 & $0,7-2,3$ \\
10 - 20 cigarrillos/día & $62 / 108$ & 2,36 & $1,4-4,1$ \\
$>$ 20 cigarrillos/día & $38 / 57$ & 2,97 & $1,5-5,8$ \\
\hline
\end{tabular}

infección latente ${ }^{53}$. De este modo, se ha planteado que la nicotina, y quizás otros componentes del tabaco, que disminuyen la producción de TNF- $\alpha$ en los macrófagos alveolares, aumentarían la susceptibilidad de los individuos a desarrollar enfermedad tuberculosa progresiva a partir de una infección por $M$. tuberculosis en estado latente $^{54,55}$.

\section{Neumonía bacteriana}

El tabaquismo activo y pasivo constituyen factores de riesgo para neumonía bacteriana (Tabla $4)^{56-59}$. La exposición prolongada a humo de tabaco favorece la colonización del tracto respiratorio y modifica la bacteriología de la neumonía adquirida en la comunidad. El consumo de tabaco asociado a enfermedad pulmonar obstructiva crónica es un factor de riesgo de neumonía indiscutible, sin embargo, el efecto del tabaquismo per se ha sido más difícil de precisar porque requiere controlar la variable confundente de la función pulmonar ${ }^{56}$. Straus y cols, examinaron los factores de riesgo de neumonía por Legionella spp, donde el tabaquismo fue un factor de riesgo independiente (OR: 3,48; IC95\%: 2,09$5,79)^{57}$. En un estudio epidemiológico, Mizooka y cols, examinaron los niveles séricos de $\operatorname{IgA}$ e IgG para Chlamydia pneumoniae en 1.063 varones y 1.573 mujeres entre 18 y 94 años, encontrando un aumento del riesgo relativo de serología positiva tipo IgA para C. pneumoniae en los fumadores (OR: 2,00; IC95\%: 1,45-2,77) ${ }^{58}$.
Hallazgos similares han sido comunicados por otros autores (OR: 5,00; IC95\%: 1,45-17,3 en hombres fumadores $)^{59,60}$. Almirall y cols, examinaron el efecto del consumo de tabaco en una cohorte de 205 adultos hospitalizados por neumonía adquirida en la comunidad, el haber fumado aumentó al doble el riesgo de neumonía (OR: 2,0; IC95\%: 1,24-3,24) y un tercio del riesgo de adquirir una neumonía en esta población fue atribuido al tabaquismo ${ }^{61}$. En un estudio poblacional realizado en Dallas, Texas, el tabaquismo se asoció a mayor riesgo de enfermedad neumocócica invasiva en adultos menores de 65 años (OR: 2,6; IC95\%: 1,9-3,5), en quienes actualmente no se recomienda la vacuna antineumocócica ${ }^{62}$. El riesgo atribuible al tabaquismo en adultos de 24-64 años fue de $31 \%$. El riesgo de enfermedad neumocócica asociado al tabaquismo muestra una fuerte relación dosis-respuesta y disminuye a los 10 años de haber suspendido el hábito tabáquico al nivel de sujetos que nunca han fumado ${ }^{27}$. El tabaquismo pasivo también aumenta el riesgo de neumonía en adultos (OR: 2,5; IC95\%: 1,2-5,1) ${ }^{27}$. En los pacientes con infección VIH y linfopenia aumenta el riesgo de neumonía comunitaria entre los fumadores ${ }^{63}$. Considerando que entre los fumadores es mayor el riesgo de colonización y neumonía por $S$. pneumoniae, $H$. influenzae y $M$. catarrhalis se ha recomendado emplear macrólidos de espectro expandido y la vacunación antineumocócica en esta categoría de riesgo ${ }^{64}$. 


\section{Neumonía por Varicela}

La Varicela es una enfermedad exantemática aguda, muy contagiosa relacionada con la estacionalidad, con alta incidencia en invierno y primavera, muy común en la infancia, que se caracteriza por brotes sucesivos de lesiones que progresan rápidamente desde las máculas y pápulas iniciales a vesículas, pústulas y costras, siendo en los niños una enfermedad leve con pocas complicaciones ${ }^{65}$. Sin embargo, cuando la contraen los adultos suele ir acompañada de fiebre elevada, notables síntomas constitucionales y complicaciones pulmonares. La neumonía provocada por varicela no es frecuente en los niños, en cambio, el 26 a 53\% de los adultos con varicela presentan evidencias clínicas o radiológicas de neumonitis durante el curso de la enfermedad ${ }^{66}$. El $25 \%$ de los decesos por varicela ocurren en adultos, especialmente debido al compromiso pulmonar, a pesar que ocasionan menos del $2 \%$ de los $\operatorname{casos}^{65}$. La mortalidad de la neumonía por varicela en adultos no tratados es cercana al $10 \%$ y se eleva sobre $50 \%$ en el grupo con falla respiratoria grave.

Varios estudios observacionales han sugerido que el riesgo de neumonía por varicela aumenta en los fumadores, lo cual ha sido atribuido a los efectos nocivos del tabaco sobre la permeabilidad del lecho capilar pulmonar y los mecanismos defensivos locales ${ }^{67-69}$. La terapia con aciclovir oral disminuye la gravedad de la infección por varicela en niños y adultos, pero se desconoce si disminuye el riesgo de desarrollar neumonitis ${ }^{70}$. El aumento de la susceptibilidad a presentar compromiso pulmonar en los sujetos fumadores, ha determinado que los expertos recomienden el uso de aciclovir en todos los pacientes adultos fumadores.

\section{Conclusión}

El tabaquismo activo y pasivo continúa siendo un problema grave de salud pública en el medio nacional y se asocia a mayor riesgo de desarrollar infecciones respiratorias, debido a los efectos nocivos de la nicotina y otros componentes del tabaco sobre la estructura y función del sistema respiratorio y el sistema inmune del huésped. Los hallazgos de esta revisión nos permiten destacar la importancia que tiene promover el cese del hábito tabáquico en la población, con el propósito de reducir el riesgo de adquirir infecciones bacterianas y virales en los individuos expuestos al humo de tabaco. Como medidas preventivas adicionales, se debería recomen- dar la vacunación antiinfluenza y antineumocócica en todos los fumadores activos que no logran abandonar el hábito.

\section{Bibliografía}

1.- WALD N J, HACKSHAW A K. Cigarette smoking: an epidemiological overview. Br Med Bull 1996; 52: 3 11.

2.- SHERMAN C B. The health consequences of cigarette smoking. Pulmonary diseases. Med Clin North Am 1992; 76: 355-75.

3.- CENTERS FOR DISEASE CONTROL. Cigarette smoking-attributable mortality and years of potential life lost - United States, 1990. MMWR 1993; 43: 1-8.

4.- DI FRANZA J R, LEW R A. Morbidity and mortality in children associated with the use of tobacco products by other people. Pediatrics 1996; 97: 560-8.

5.- MURIN S, BILELLO K S, MATTHAY R. Other smoking-affected pulmonary diseases. Clin Chest Med 2000; 21: 121-37.

6.- AUBRY M C, WRIGHT J L, MYERS J L. The pathology of smoking-related lung diseases. Clin Chest Med 2000; 21: 11-35.

7.- FOSTER W M, LANGENBACK E G, BERGOFSKY E $H$. Disassociation in the mucociliary function of central and peripheral airways of asymptomatic smokers. Am Rev Respir Dis 1985; 132: 633-9.

8.- ROBERTS C M, CAIRNS D, BRYANT D H, BURKE W M, YEATES M, BLAKE $\mathrm{H}$, et al. Changes in epithelial lining fluid albumin associated with smoking and interstitial lung disease. Eur Respir J 1993; 6: $110-5$.

9.- $\quad$ SUNDRAM F X. Clinical studies of alveolar-capillary permeability using technetium-99m DTPA aerosol. Ann Nucl Med 1995; 9: 171-8.

10.- BAL COOPERATIVE GROUP STEERING COMMITTEE. Bronchoalveolar lavage constituents in healthy individuals, idiopathic pulmonary fibrosis and selected comparison groups. Am Rev Respir Dis 1990; 141: S169-202.

11.- KUSCHNER W G, D'ALESSANDRO A, WONG H, BLANC P D. Dose-dependent cigarette smoking-related inflammatory responses in healthy adults. Eur Respir J 1996; 9: 1989-94.

12.- HOCKING W G, GOLDE D W. The pulmonaryalveolar macrophage (first of two parts). N Engl J Med 1979; 301: 580-7.

13.- BROWN G P, IWAMOTO G K, MONICK M M, HUNNINGHAKE G W. Cigarette smoking decreases interleukin-1 release by human alveolar macrophages. Am J Physiol 1989; 256: C260-64.

14.- LAUGHTER A H, MARTIN R R, TWOMEY J J. Lymphoproliferative responses to antigens mediated by human pulmonary alveolar macrophages. J Lab Clin Med 1977; 89: 1326-32.

15.- HOGG J C. Felix Fleischner Lecture. The traffic of polymorphonuclear leukocytes through pulmonary microvessels in health and disease. Am J Roentgenol 1994; 163: 769-75.

16.- DANIELE R P, DAUBER J H, ALTOSE M D, ROWLANDS D T Jr, GORENBERG D J. Lymphocyte studies in asymptomatic cigarette smokers. A comparison between lung and peripheral blood. Am Rev Respir Dis 1977; 116: 997-1005. 
17.- TERASHIMA T, WIGGS B, ENGLISH D, HOGG J C, VAN EEDEN S F. The effect of cigarette smoking on the bone marrow. Am J Respir Crit Care Med 1997; 155: 1021-6.

18.- TOLLERUD D J, CLARK J W, BROWN L M, NEULAND C Y, MANN D L, PANKIW-TROST L K, et al. The effects of cigarette smoking on T-cell subsets. A population-based survey of healthy caucasians. Am Rev Respir Dis 1989; 139: 1446-51.

19.- PARK C S, OLIVER L C, RENZI P M, et al. Natural killer cell activity in smokers: characterization of the defect and response to biological modifiers. Am Rev Respir Dis 1987; 135: 154A.

20.- TRABER M G, VAN DER VLIET A, REZNICK A Z, CROSS C E. Tobacco-related diseases. Is there a role for antioxidant micronutrient supplementation? Clin Chest Med 2000; 21: 173-87.

21.- MARANGON K, HERBETH B, LECOMTE E, PAULDAUPHIN A, GROLIER P, CHANCERELLE Y, et al. Diet, antioxidant status, and smoking habits in French men. Am J Clin Nutr 1998; 67: 231-9.

22.- ADAIR-BISCHOFF C E, SAUVE R S. Environmental tobacco smoke and middle ear disease in preschoolage children. Arch Pediatr Adolesc Med 1998; 152: 127-33.

23.- GÜRKAN F, KIRAL A, DAGLI E, KARAKOC F. The effect of passive smoking on the development of respiratory syncytial virus bronchiolitis. Eur J Epidemiol 2000; 16: 465-8.

24.- STRACHAN D P, COOK D G. Health effects of passive smoking. 1. Parental smoking and lower respiratory illness in infancy and early childhood. Thorax 1997; 52: 905-14.

25.- JEDRYCHOWSKI W, FLAK E. Maternal smoking during pregnancy and postnatal exposure to environmental tobacco smoke as predisposition factors to acute respiratory infections. Environ Health Perspect 1997; 105: 302-6.

26.- LIPSKY B A, BOYKO E J, INUI T S, KOEPSELL T D. Risk factors for acquiring pneumococcal infections. Arch Intern Med 1986; 146: 2179-85.

27.- NUORTI J P, BUTLER J C, FARLEY M M, HARRISON L H, McGEER A, KOLCZAK M S, et al. Cigarette smoking and invasive pneumococcal disease. Active Bacterial Core Surveillance Team. N Engl J Med 2000; 342: 681-9.

28.- DYE J A, ADLER K B. Effects of cigarette smoke on epithelial cells of the respiratory tract. Thorax 1994; 49: 825-34.

29.- PABST M J, PABST K M, COLLIER J A, COLEMAN T C, LEMONS-PRINCE M L, GODAT M S, et al. Inhibition of neutrophil and monocyte defensive functions by nicotine. J Periodontol 1995; 66: $1047-$ 55.

30.- BRIDGES R B, KRAAL J H, HUANG L J, CHANCELLOR B M. Effects of tobacco smoke on chemotaxis and glucose metabolism of polymorphonuclear leukocytes. Infect Immun 1977; 15: 11523.

31.- NUMABE Y, OGAWA T, KAMOI H, KIYONOBU K, SATO S, KAMOI K, et al. Phagocytic function of salivary PMN after smoking or secondary smoking. Ann Periodontol 1998; 3: 102-7.

32.- GINNS L C, GOLDENHEIM P D, MILLER L G, BURTON R C, GILLICK L, COLVIN R B, et al. Tlymphocyte subsets in smoking and lung cancer: analysis of monoclonal antibodies and flow cytometry. Am Rev Respir Dis 1982; 126: 265-9.
33.- HOLT P G. Immune and inflammatory function in cigarette smokers. Thorax 1987; 42: 241-9.

34.- MERRILL W W, GOODENBERGER D, STROBER W, MATTHAY R A, NAEGEL G P, REYNOLDS H Y. Free secretory component and other proteins in human lung lavage. Am Rev Respir Dis 1980; 122: 156-61.

35.- PETERS J M, FERRIS B G. Smoking, pulmonary function, and respiratory symptoms in a college-age group. Am Rev Respir Dis 1967; 95: 774-82.

36.- BOAKE W C. A study of illness in a group of Cleveland families. XVIII. Tobacco smoking and respiratory infections. N Engl J Med 1958; 259: 1245-9.

37.- ARONSON M D, WEISS S T, BEN R L, KOMAROFF A L. Association between cigarette smoking and acute respiratory tract illness in young adults. JAMA 1982; 248: 181-3.

38.- COHEN S, TYRREL D A, RUSSELL M A, JARVIS M J, SMITH A P. Smoking, alcohol consumption, and susceptibility to the common cold. Am J Public Health 1993; 83: 1277-83.

39.- COOK D G, STRACHAN D P. Health effects of passive smoking-10: summary of effects of parental smoking on the respiratory health of children and implications for research. Thorax 1999; 54: 357-66.

40.- EY J L, HOLBERG C J, ALDOUS M B, WRIGHT A L, MARTINEZ F D, TAUSSIG L M. Passive smoke exposure and otitis media in the first year of life. Group Health Medical Associates. Pediatrics 1995; 95: 670-7.

41.- RICHARDSON M A. Upper airway complications of cigarette smoking. J Allergy Clin Immunol 1988; 81: 1032-5.

42.- ARBES S J, AGUSTSDOTTIR H, SLADE G D. Environmental tobacco smoke and periodontal disease in the United States. Am J Public Health 2001; 91: 253-7.

43.- MULLER H P. Smoking and periodontal health. Gesundheitswesen 2000; 62: 400-8.

44.- ROGOT E, MURRAY J L. Smoking and causes of death among U.S. veterans: 16 years of observation. Public Health Rep 1980; 95: 213-22.

45.- KARK J D, LEBIUSH M, RANNON L. Cigarette smoking as a risk factor for epidemic $\mathrm{A}(\mathrm{H} 1 \mathrm{~N} 1)$ influenza in young men. N Engl J Med 1982; 307: 1042-6.

46.- FINKLEA J F, SANDIFER S H, SMITH D D. Cigarette smoking and epidemic influenza. Am J Epidemiol 1969; 90: 390-9.

47.- FINKLEA J F, HASSELBLAD V, RIGGAN W B, NELSON W C, HAMMER D I, NEWILL V A. Cigarette smoking and hemagglutination inhibition response to influenza after natural disease and immunization. Am Rev Respir Dis 1971; 104: 36876.

48.- ANDERSON R H, SY F S, THOMPSON S, ADDY C. Cigarette smoking and tuberculin skin test conversion among incarcerated adults. Am J Prev Med 1997; 13 : 175-81.

49.- ALTET M N, ALCAIDE J, PLANS P, TABERNER J L, SALTO E, FOLGUERA L I, et al. Passive smoking and risk of pulmonary tuberculosis in children immediately following infection. A case-control study. Tuber Lung Dis 1996; 77: 537-44.

50.- ALCAIDE J, ALTET M N, PLANS P, PARRON I, FOLGUERA L, SALTO E, et al. Cigarette smoking as a risk factor for tuberculosis in young adults: a casecontrol study. Tuber Lung Dis 1996; 77: 112-6.

51.- DAVIES P D, YEW W W, GANGULY D, DAVIDOW A L, REICHMAN L B, DHEDA K, et al. Smoking and 
tuberculosis: the epidemiological association and immunopathogenesis. Trans R Soc Trop Med Hyg 2006; 100: 291-8.

52.- GAJALAKSHMI V, PETO R, KANAKA T S, JHA P. Smoking and mortality from tuberculosis and other diseases in India: retrospective study of 43000 adult male deaths and 35000 controls. Lancet 2003; 362: 507-15.

53.- KEANE J, GERSHON S, WISE R P, MIRABILELEVENS E, KASZNICA J, SCHWIETERMAN W D, et al. Tuberculosis associated with infliximab, a tumor necrosis factor alpha-neutralizing agent. N Engl J Med 2001; 345: 1098-104.

54.- FLOTO R A, SMITH K G. The vagus nerve, macrophages, and nicotine. Lancet 2003; 361: 1069-70.

55.- WANG $\mathrm{H}, \mathrm{YU} \mathrm{M}$, OCHANI M, AMELLA C A, TANOVIC M, SUSARLA S, et al. Nicotinic acethylcholine receptor alpha7 subunit is an essential regulator of inflammation. Nature 2003; 421: 384-8.

56.- LANGE P, VESTBO J, NYBOE J. Risk factors for death and hospitalization from pneumonia. A prospective study of a general population. Eur Respir J 1995; 8: 1694-8.

57.- STRAUS W L, PLOUFFE J F, FILE TM Jr, LIPMAN H B, HACKMAN B H, SALSTROM S J, et al. Risk factors for domestic acquisition of Legionnaires disease. Ohio Legionnaires Disease Group. Arch Intern Med 1996; 156: 1685-92.

58.- MIZOOKA M, ISHIKAWA S, J M S Cohort Study Group. Prevalence of Chlamydia pneumoniae in Japanese rural districts; association of smoking and physical activity with Chlamydia pneumoniae seropositivity. Intern Med 2003; 42: 960-6.

59.- KARVONEN M, TUOMILEHTO J, PITKÄNIEMI J, NAUKKARINEN A, SAIKKU P. Importance of smoking for Chlamydia pneumoniae seropositivity. Int $\mathrm{J}$ Epidemiol 1994; 23: 1315-21.
60.- VON HERTZEN L, KAPRIO J, KOSKENVUO M, ISOAHO R, SAIKKU P. Humoral immune response to Chlamydia pneumoniae in twin discordant for smoking. J Intern Med 1998; 244: 227-34.

61.- ALMIRALL J, GONZÁLEZ CA, BALANZO X, BOLIBAR I. Proportion of community-acquired pneumonia cases attributable to tobacco smoking. Chest 1999; 116: 375-9.

62.- PASTOR P, MEDLEY F, MURPHY T V. Invasive pneumococcal disease in Dallas County, Texas: results from population-based surveillance in 1995. Clin Infect Dis 1998; 26: 590-5.

63.- CONLEY L J, BUSH T J, BUCHBINDER S P, PENLEY K A, JUDSON F N, HOLMBERG S D. The association between cigarette smoking and selected HIV-related medical conditions. AIDS 1996; 10: 11216.

64.- BARTLETT J G, BREIMAN R F, MANDELL L A, FILE T M. Community-acquired pneumonia in adults: guidelines for management. The Infectious Diseases Society of America. Clin Infect Dis 1998; 26: 81138 .

65.- KAUFMAN R S. Viral pneumonia. In Pennington JE (ed): Respiratory Infections: Diagnosis and Management. New York, Raven, 1983, pp 402-7.

66.- FELDMAN S. Varicella-zoster virus pneumonitis. Chest 1994; 106: 22S-27S.

67.- ELLIS M E, NEAL K R, WEBB A K. Is smoking a risk factor for pneumonia in adults with chickenpox? Br Med J (Clin Res Ed) 1987; 294: 1002.

68.- GRAYSON M L, NEWTON-JOHN H. Smoking and varicella pneumonia [letter]. J Infect 1988; 16: 312.

69.- OH H M, CHEW S K. Varicella pneumonia in adults clinical spectrum. Ann Acad Med Singapore 1996; 25 : 816-9.

70.- ARVIN A M. Varicella-zoster virus. Clin Microbiol Rev 1996; 9: 361-81.

Correspondencia a:

Dr. Fernando Saldías Peñafiel

Departamento de Enfermedades Respiratorias

Pontificia Universidad Católica de Chile

Teléfonos: (562) 6331541 - (562) 3543242

FAX: (562) 6335255

Marcoleta 352

Santiago, Chile

E-mail: fsaldias@med.puc.cl 